\title{
Interference Alignment Based Transceiver Design in OSG mode of HetNets
}

\author{
Qin Niu, Zhimin Zeng, Tiankui Zhang and Zhirui Hu \\ Beijing Key Laboratory of Network System Architecture and Convergence, \\ Beijing University of Posts and Telecommunications Beijing, China \\ [e-mail: aniunq001@bupt.edu.cn, tkzhang@gmail.com] \\ *Corresponding author: Tiankui Zhang
}

Received December 15, 2014; revised March 18, 2015; accepted May 3, 2015; published June 30, 2015

\begin{abstract}
This paper focuses on solving co-channel interference (CCI) issues arising in the open subscriber group (OSG) mode of heterogeneous networks (HetNets). Considering a general framework consisting of arbitrary number of picocells within a macro cell, where the inter-user interference (IUI) is the main CCI to macro user equipments (UEs), while the the inter-cell interference (ICI) is the major CCI to pico UEs. In this paper, three IA based transceiver design schemes are proposed. For macro cell, we uniformly use block diagonalization (BD) scheme to eliminate the IUI. And for picocells, three IA schemes are proposed to mitigate the ICI. The first scheme, named as zero forcing IA (ZF-IA) scheme, aligns the inter picocell interference onto an arbitrary sub-space of the cross-tier interference using ZF scheme. Considering the channel state information (CSI) of the desired channel of pico UEs, the second scheme, named as optimal desired sub-channel selected IA (ODC-IA) scheme, aligns the inter picocell interference onto a certain sub-space of the cross-tier interference, which guarantees the largest channel gain of the desired signal of pico UEs. The third IA scheme, named as maximum cross-tier interference selected IA (MI-IA) scheme, is designed for the system with less receive antennas. The inter picocell interference is aligned onto the space of the strongest cross-tier interference and only the interference on this space is nullified. The complexity analysis and simulations show that the proposed transceiver design schemes outperform the existing IA schemes in the OSG mode of HetNets, and the MI-IA scheme reduces the requirement of the receive antennas number with lower complexity.
\end{abstract}

Keywords: Heterogeneous networks; open subscriber group; transceiver design; interference alignment

This work was supported by the Special Funding for Beijing Common Construction Project and Beijing Natural Science Foundation (4144079) 


\section{Introduction}

Heterogeneous networks (HetNets) are considered as one of the most promising techniques to meet the explosively increasing traffic demand in wireless networks. By deploying a number of small cells, such as picocells and femtocells, overlaid with conventional macro networks, the coverage is extended and the spectral utilization is increased. Nevertheless, as the macrocell and small cells reuse the same spectrum resources, the user equipments (UEs) experience severe co-channel interference (CCI), especially in small cell enhanced scenario where small cells are deployed nearby. The CCI seriously hinders the spatial reuse gain brought by small cells and is believed as the major bottleneck to data rate improvement. In addition, as the cell density increases, classical resource management techniques based on frequency/space reuse and power control are unable to cope with the additional interference effectively. Therefore, various interference management schemes have been considered in both academia and industry field[1-4]. As an advanced beamforming technique, interference alignment (IA), not only achieving higher capacity than the previously believed but also reducing the system complexity, has been investigated as an effective interference management technique. IA refers to the determination of beamforming directions in such a manner that the interference from other transmitters is aligned, rather than cancel or reduce, in specific signal dimensions separated from the desired signals at their respective receivers[5].

IA is pioneered in multi-user multiple input multiple output (MU-MIMO) interference channel [5-8]. It concluded that each user can use half of the channel resources to make an interference-free communication regardless of user number. According to the process of solving precoding matrices, IA can be devided into two branches. One is the linear IA algorithms in closed-form expressions [5, 6], and the other is the iterative IA algorithms utilizing the reciprocity of wireless channel in cellular networks [7, 8]. Recently, IA has been employed to manage the interference in conventional single-tier cellular networks $[9,10]$ and two-tier HetNets [3-4], [11-20]. [11] extends the traditional IA scheme [5] to HetNets aiming at mitigating the co-tier interference. Two hierarchical IA (HIA) schemes are proposed for the principal interference scenarios, i.e., the closed subscriber group (CSG) mode and the open subscriber group (OSG) mode [3], respectively. Then, the system level performance of HIA scheme is investigated in multi-cell environments [4]. The above schemes [3, 4, 11] design the precoding matrices for the pico base stations (BSs) via solving the generalized eigen-problem, constraining them only be applied to some specific networks, such as the network with two or three picocells in a macrocell, and cannot be straightforwardly extended to general case with arbitrary number of picocells and macro UEs in a macrocell. In the general case of HetNets, the IA schemes are proposed for the CSG mode or the system which is equivalent to (or can be simplified to) the CSG mode [12-20]. The IA scheme in [12, 13] aims to mitigate the co-tier interference by formulate a coalitional game in partition form among the small cells, but it does not deal with the cross-tier interference. The IA scheme in $[14,15]$ removes the cross-tier interference using a distributed algorithm, yet the co-tier interference is not addressed. Taking both of the co-tier and cross-tier interference into consideration, an extended hierarchical IA (EHIA) scheme [16], a ZF based transceiver design scheme [17], a IA and bit allocation scheme [18] and some multi-stage IA schemes [19-21] are proposed in closed form expressions. The hierarchical multi-stage IA scheme in [19] obtains the precoding matrices by dividing the aligning process into multiple stages, while the two-stage IA schemes proposed in [20] and [21] model the HetNets as a partially connected interference channel with two 
sub-systems. One sub-system which is only influenced by the cross-tier interference is the focus of design, and the conventional IA is carried out in the other subsystem which is impacted by both co-tier and cross-tier interference.

To the best of our knowledge, there are few IA schemes specifically for the general case of OSG mode so far. In the OSG mode, the interference is different from that in the CSG mode. That is, the cross-tier interference from pico BSs to macro UEs, which cannot be neglected in the CSG mode, is negligible in the OSG mode owing to the cell range expansion (CRE) strategy [3]. So if the IA schemes proposed for the CSG mode is employed in the OSG mode, there must be some costs to deal with the neglected interference, thus the performance is unsatisfactory. Therefore, it is necessary to design new schemes dedicated to OSG mode.

In this paper, three IA based transceiver design schemes are proposed in the OSG mode of downlink HetNets. In these schemes, the inter macro-user interference is eliminated by the precoding matrix for macro BS and the interference suppression matrices for macro UEs, meanwhile the cross-tier interference and the inter picocell interference are mitigated by the precoding matrices of the pico BSs and the interference suppression matrices of pico UEs.

1) The first IA based transceiver design scheme aligns the inter picocell interference onto an arbitrary sub-space of the cross-tier interference using ZF scheme, which is named as ZF-IA scheme.

2) ZF-IA scheme works effectively, yet it has not considered the channel state information (CSI) of the desired channel in the alignment processing, that is, if we take this information into account, the data rate can be further improved. So we then propose the second IA based transceiver design scheme, which is named as optimal desired sub-channel selected IA (ODC-IA) scheme. In the process of designing the precoding matrices of the pico BSs, we align the inter picocell interference onto a certain sub-space of the cross-tier interference, ensuring that the desired signal for pico UEs experience spatial subchannels with better CSI.

3) ZF-IA scheme and ODC-IA scheme provide higher data rate. While considering the system with less receive antennas, we finally propose the third IA based transceiver design scheme, named as maximum cross-tier interference selected IA (MI-IA) scheme, to reduce the requirement of receive antennas number, in which we align the inter picocell interference onto the space of the strongest cross-tier interference, and then nullify the aligned interference only.

4) It can be seen from the complexity analysis and simulation results that the proposed transceiver design schemes can achieve larger data rate than the existing IA schemes in the OSG mode of downlink HetNets. In addition, not only requirement of receive antennas number but also complexity can be reduced validly with the MI-IA scheme, while a little gain of data rate has to be paid as the cost.

The remainder of this paper is organized as follows. Section 2 describes the system model for the OSG mode of downlink two-tier HetNets. In section 3, three IA based transceiver design scheme are proposed to mitigate CCI, further improve the performance or reduce the overhead. Finally, the complexity analysis and simulations are given in section 4 and conclusions are drawn in section 5 , respectively.

Notation: matrices and vectors are denoted by boldface upper case and boldface lower case symbols, respectively. I represents the identity matrix and $\mathbf{0}$ represents the all-zero matrix. $(\mathbf{A})^{\mathrm{T}},(\mathbf{A})^{\mathrm{H}},\|\mathbf{A}\|_{2}, \operatorname{span}(\mathbf{A}), \operatorname{null}(\mathbf{A})$ and $\mathbf{A}^{\dagger}=\mathbf{A}^{\mathrm{H}}\left(\mathbf{A} \mathbf{A}^{\mathrm{H}}\right)^{-1}$ indicate transpose, Hermitian, the Frobenius norm, the column space, the null space and the Moore-Penrose inverse matrix of $\mathbf{A}$, respectively. $\mathbf{A} \prec \mathbf{B}$ means that the column space of $\mathbf{A}$ is included in that of $\mathbf{B}$. 


\section{System Model}

Consider the OSG mode of downlink two-tier HetNets with $L+1$ cells as shown in Fig. 1, which consists of a single macro cell and $L$ picocells. The picocells are overlapped with the macro cell and all cells use the same carrier frequency. We concern with the scenarios in which the picocells are adjacent to each other and have strong mutual interference. For convenience, the macro BS is denoted by MBS $M$, and $L$ pico BSs are indexed by PBS $l \in\{1, \cdots, L\}$.The macro BS serves $K$ macro UEs (indexed by MUE $k \in\{L+1, \cdots, L+K\}$ ) simultaneously and each pico BS serves one pico UE (indexed by PUE $l \in\{1, \cdots, L\}$ ). In this system, the macro BS is equipped with $N_{M T}$ transmitting antennas, each pico BS is equipped with $N_{P T}$ transmitting antennas uniformly, and each UE is equipped with $N_{R}$ receive antennas uniformly. We assume that each UE receives $d$ independent streams from its corresponding BS, and each pico BS exchanges only control messages, such as CSI [22], to the macro BS over the backhaul link.

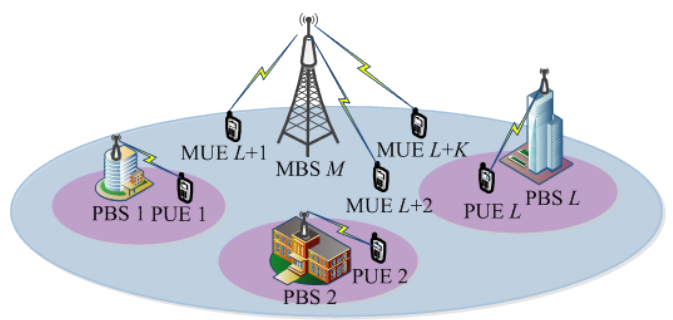

Fig. 1. Downlink two-tier HetNets

In OSG mode of HetNets, a CRE strategy [23] is utilized to extend the coverage area of pico BS as shown in Fig. 2, by which the cross-tier interference from the pico BSs to the macro UEs can be negligible [3]. In the conventional cell selection mechanism, each UE selects its serving cell from which the largest reference signal received power (RSRP) is provided [23, 24]. In the CRE strategy, an offset is added to the RSRP in the conventional cell selection mechanism. As a consequence, the cell border level will be the addition of the RSRP and the offset, i.e., in the CRE area, $\gamma_{M} \leq \gamma_{P}+\boldsymbol{\alpha}$, where $\gamma_{M}$ and $\gamma_{P}$ are the RSRP measured from the macro BS and the pico BS, respectively; $\boldsymbol{\alpha}$ is CRE offset. Yet, the UEs in this area are also served by the pico BS. Meanwhile, in the macro coverage area, the RSRP measured from the macro BS is larger than that measured from the pico BS, i.e., $\gamma_{M}>\gamma_{P}+\alpha$. A lot of researches on setting the value of $\boldsymbol{\alpha}$ have been investigated [25, 26], to the best of our knowledge, there has not been a uniform standard yet. The cross-tier interference from the pico BSs to the macro UEs decrease along with $\boldsymbol{\alpha}$, while the pico UEs with large $\boldsymbol{\alpha}$ can suffer from severe cross-tier interference. This paper focuses on the large $\boldsymbol{\alpha}$ case, in which the cross-tier interference from the pico BSs to macro UEs can be negligible.

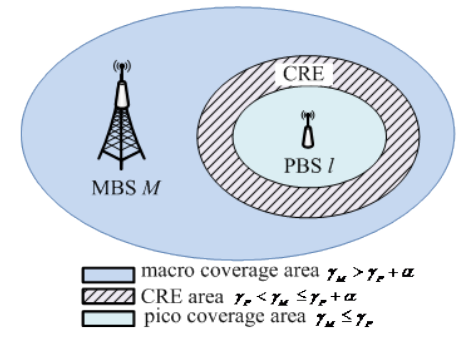

Fig. 2. CRE area illustration 


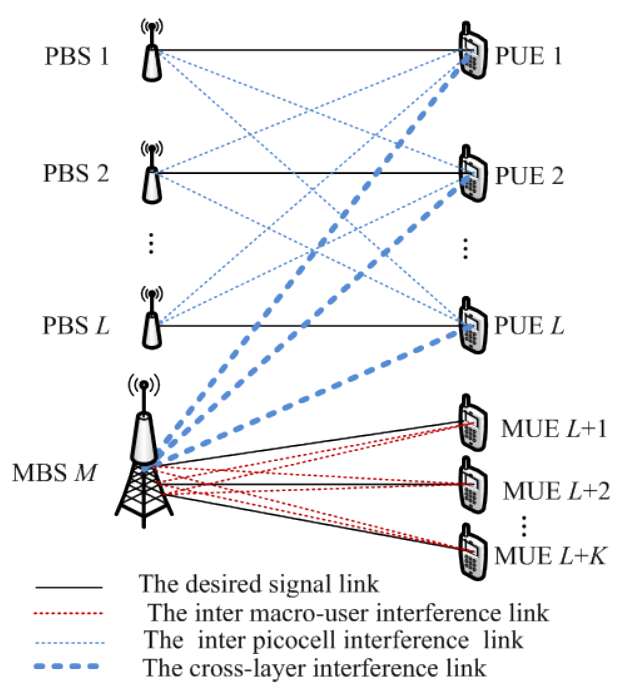

Fig. 3. System model for the OSG mode of downlink two-tier HetNets

The system model for the OSG mode of downlink two-tier HetNets is as shown in Fig. 3. With CRE strategy, the inter macro-user interference is the main interference to macro UEs, and the received signal at $\operatorname{UE} k(\forall k=\{L+1, \cdots, L+K\})$ is

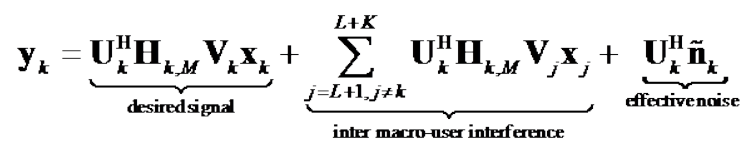

where $\mathbf{x}_{\boldsymbol{k}} \in C^{d \times 1}$ denotes the transmitted symbol to UE $k . \mathbf{V}_{k} \in C^{N_{\mathrm{MT}} \times d}$ represents the precoding matrix for carrying $\mathbf{x}_{\boldsymbol{k}}$, which is normalized such that $\mathbf{V}_{\boldsymbol{k}} \mathbf{V}_{\boldsymbol{k}}^{\mathbf{H}}=\mathbf{I}$. The transmit signal of BS $M$ satisfies an average power constraint, i.e., $E\left(\sum_{k=L+1}^{L+K}\left\|\mathbf{V}_{k} \mathbf{x}_{k}\right\|_{2}^{2}\right) \leq P_{M}$, where $P_{M}$ is the average power of BS $M$. $\mathbf{H}_{k, M} \in C^{N_{\mathbb{R}} \times N_{M T}}$ means the channel matrix from BS $M$ to UE $k$. $\mathbf{U}_{\boldsymbol{k}}^{\mathrm{H}} \in C^{d \times N_{R}}$ indicates the interference suppression matrix of UE $k$, which is normalized such that $\mathbf{U}_{\boldsymbol{k}} \mathbf{U}_{\boldsymbol{k}}^{\mathbf{H}}=\mathbf{I}$. $\tilde{\mathbf{n}}_{\boldsymbol{k}} \in C^{N_{R} \times 1}$ is the effective noise which includes the cross-tier interference from the pico BSs to the macro UEs and the received noise $\mathbf{n}_{\boldsymbol{k}} \in \boldsymbol{C}^{\boldsymbol{N}_{\mathbf{R}} \times \mathbf{1}}$, where $\mathbf{n}_{\boldsymbol{k}}$ is a zero mean complex Gaussian noise vector with independently distributed entries of unit variance each.

The pico UEs are only interfered by the inter-cell interference (ICI) which contains the inter picocell interference and the cross-tier interference from the macro BS. The received signal at $\mathrm{UE} l(\forall l=\{1, \cdots, L\})$ is represented as

$$
\mathbf{y}_{l}=\underbrace{\mathbf{U}_{l}^{\mathrm{H}} \mathbf{H}_{l} \mathbf{V}_{l} \mathbf{x}_{l}}_{\text {desired sgnal }}+\underbrace{\sum_{j=1, j \neq l}^{L} \mathbf{U}_{l}^{\mathrm{H}} \mathbf{H}_{l, j} \mathbf{V}_{j} \mathbf{x}_{j}+\sum_{\mathbf{k}=L+1}^{L+K} \mathbf{U}_{l}^{\mathrm{H}} \mathbf{H}_{l, M} \mathbf{V}_{\mathbf{k}} \mathbf{x}_{\mathbf{k}}}_{\text {ICI }}+\underbrace{\mathbf{U}_{l}^{\mathrm{H}} \mathbf{n}_{l}}_{\text {noise }}
$$

The definition of the relevant parameters corresponding to the picocell in (2) is similar to that of the macrocell in (1). 


\section{The Proposed Transceiver Design Schemes}

In this section, we propose three IA based transceiver design schemes in the OSG mode of downlink HetNets. The proposed schemes consist of two parts, the transceiver design for macro cell and the transceiver design for picocells. For macro cell, we uniformly use the block diagonalization (BD) scheme to eliminate the inter macro-user interference. On the contrary, different IA schemes are employed to mitigate the cross-tier interference and the inter picocell interference for picocells.

\subsection{Transceiver design for macro cell}

The proposed transceiver design for macro cell can be summarized as follows: Firstly, the inter macro-user interference is removed by the precoding matrix of the macro BS, and then the inter-stream interference is mitigated by interference suppression matrices of macro UEs.

1) Design of the precoding matrix for the macro BS

There are different approaches in the literatures to remove the inter macro-user interference to macro UEs. In this section, we design the precoding matrix of the macro BS based on BD scheme owning to its simplicity and validity. Therefore, the precoding matrix of the macro BS for macro UE $k$ ( $\forall k \in\{L+1, \cdots, L+K\})$ is designed according to the following decomposition:

$$
\mathbf{V}_{k}=\mathbf{V}_{k}^{0} \mathbf{V}_{k}^{1}
$$

where $\mathbf{V}_{k}^{0}$ is used to eliminate the interference from the non-intended macro UEs and $\mathbf{V}_{k}^{1}$ is the matrix to optimize the transmission. In order to cancel all the inter macro-user interference, the following constraint must be satisfied

$$
\mathbf{H}_{k, M} \mathbf{V}_{j}=\mathbf{0}, \forall j \in\{L+1, \cdots, L+K\}, j \neq k
$$

Thus $\mathbf{V}_{k}$ should lie in the null space of $\overline{\mathbf{H}}_{k, M}$, i.e., $\mathbf{V}_{k} \subset \operatorname{null}\left(\overline{\mathbf{H}}_{k, M}\right)$, where $\overline{\mathbf{H}}_{k, M}$ is defined as

$$
\overline{\mathbf{H}}_{k, M}=\left[\begin{array}{llllll}
\mathbf{H}_{L+1, M}^{\mathrm{T}} & \cdots & \mathbf{H}_{L+k-1, M}^{\mathrm{T}} & \mathbf{H}_{L+k+1, M}^{\mathrm{T}} & \cdots & \mathbf{H}_{L+K, M}^{\mathrm{T}}
\end{array}\right]^{\mathrm{T}}
$$

The singular value decomposition (SVD) of $\overline{\mathbf{H}}_{k, M}$ is

$$
\overline{\mathbf{H}}_{k, M}=\overline{\mathbf{U}}_{k, M}\left[\begin{array}{cc}
\overline{\boldsymbol{\Sigma}}_{k, M} & \mathbf{0} \\
\mathbf{0} & \mathbf{0}
\end{array}\right]\left[\left(\overline{\mathbf{V}}_{k, M}\right)^{(1)}\left(\overline{\mathbf{V}}_{k, M}\right)^{(0)}\right]^{\mathrm{H}}
$$

where $\overline{\boldsymbol{\Sigma}}_{k, M}$ is a diagonal matrix with singular values of $\overline{\mathbf{H}}_{k, M} \cdot\left(\overline{\mathbf{V}}_{k, M}\right)^{(1)}$ consists of the singular vectors corresponding to non-zero singular values and $\left(\overline{\mathbf{V}}_{k, M}\right)^{(0)}$ consists of the singular vectors corresponding to zero singular values. Thus the column vector of $\left(\overline{\mathbf{V}}_{k, M}\right)^{(0)}$ is an orthogonal basis for the null space of $\overline{\mathbf{H}}_{k, M}$. To guarantee the null space of $\overline{\mathbf{H}}_{k, M}$ exists, $N_{M T}>(K-1) N_{R}$ should be satisfied. By setting $\mathbf{V}_{k}^{0}=\left(\overline{\mathbf{V}}_{k, M}\right)^{(0)}$, we can guarantee that (4) is always fulfilled. Then, the inter macro-user interference to macro UEs is removed and the 
multi-user (MU) MIMO channel is equal to $K$ parallel single-user (SU) MIMO channel with no interference among them. Each equivalent SU MIMO channel $\mathbf{H}_{k, M} \mathbf{V}_{k}^{0}$ is processed separately to optimize the transmission as follows. Define the SVD of $\mathbf{H}_{k, M}\left(\overline{\mathbf{V}}_{k, M}\right)^{(0)}$ as

$$
\mathbf{H}_{k, M}\left(\overline{\mathbf{V}}_{k, M}\right)^{(0)}=\mathbf{U}_{k, M}\left[\begin{array}{cc}
\boldsymbol{\Sigma}_{k, M} & \mathbf{0} \\
\mathbf{0} & \mathbf{0}
\end{array}\right]\left[\left(\mathbf{V}_{k, M}\right)^{(1)}\left(\mathbf{V}_{k, M}\right)^{(0)}\right]^{\mathrm{H}}
$$

where $\left(\mathbf{V}_{k, M}\right)^{(1)}$ is used to maximize the received signal-to-interference-plus-noise ratio (SINR) of UE $k$. In order to choose the channel with best quality for UE $k$ to receive $d$ independent streams, we set $\mathbf{V}_{k}^{1}=\left(\mathbf{V}_{k, M}\right)^{(1)}\left(\begin{array}{c}\mathbf{I}_{d} \\ \mathbf{0}\end{array}\right)$. So the precoding matrix of UE $k$ is designed as

$$
\mathbf{V}_{k}=\left(\overline{\mathbf{V}}_{k, M}\right)^{(0)}\left(\mathbf{V}_{k, M}\right)^{(1)}\left(\begin{array}{c}
\mathbf{I}_{d} \\
\mathbf{0}
\end{array}\right)
$$

Then the precoding matrix of BS M is $\mathbf{V}_{M}=\left[\mathbf{V}_{L+1}, \cdots, \mathbf{V}_{L+K}\right]$. For the reason that $\left(\overline{\mathbf{V}}_{k, M}\right)^{(0)}$ is a $N_{M T} \times\left[N_{M T}-(K-1) N_{R}\right]$ matrix, and then $\mathbf{H}_{k, M}\left(\overline{\mathbf{V}}_{k, M}\right)^{(0)}$ is a $N_{R} \times\left[N_{M T}-(K-1) N_{R}\right]$ matrix. Thus to ensure $\mathbf{H}_{k, M}\left(\overline{\mathbf{V}}_{k, M}\right)^{(0)}$ is a full row rank matrix, $N_{M T} \geq K N_{R}$ should be satisfied.

2) Design of the interference suppression matrices for macro UEs

The inter macro-user interference to macro UEs is removed, so the matrix $\left[\mathbf{H}_{L+1, M}^{\mathrm{T}}, \cdots, \mathbf{H}_{L+K, M}^{\mathrm{T}}\right]^{\mathrm{T}} \mathbf{V}_{M}$ is block diagonal. In order to nullify the inter-stream interference, the interference suppression matrix can be designed as the Hermitian of the left singular vector matrix of $\mathbf{H}_{k, M}\left(\overline{\mathbf{V}}_{k, M}\right)^{(0)}$, i.e.,

$$
\mathbf{U}_{k}^{\mathrm{H}}=\mathbf{U}_{k, M}^{\mathrm{H}}
$$

Then, the matrix $\left[\left(\mathbf{U}_{L+1}^{\mathrm{H}} \mathbf{H}_{L+1, M}\right)^{\mathrm{T}}, \cdots,\left(\mathbf{U}_{L+K}^{\mathrm{H}} \mathbf{H}_{L+1, K}\right)^{\mathrm{T}}\right]^{\mathrm{T}} \mathbf{V}_{M}$ is diagonal and the capacity of macro UEs is maximized.

Let us add a comment stating that in the proposed transceiver design schemes, all of the matrices are designed at the macro BS side, and then transmitted to each corresponding pico BS (or UE) by the backhaul link (or the downlink channel). The procedure of the proposed Transceiver design for macro cell is shown in Table 1.

Table 1. The procedure of the proposed transceiver design for macro cell

Step 1. The macro UE $k(\forall k \in\{L+1, \cdots, L+K\}$ ) estimates the desired CSI, i.e.,

$\mathbf{H}_{k, M}$, using pilots or a preamble, then they feed back the CSI to the macro BS through a dedicated common control channel.

Step 2. The precoding matrix for the macro BS is designed using (8) and $\mathbf{V}_{M}=\left[\mathbf{V}_{L+1}, \cdots, \mathbf{V}_{L+K}\right]$. 
Step 3. The interference suppression matrices for macro UEs are designed according to (9).

Step 4. The interference suppression matrices for macro UEs are transmitted from the macro BS to each corresponding macro UE by the downlink channel.

\subsection{Transceiver design for picocells}

In this section, we proposed three IA schemes, named ZF-IA scheme, ODC-IA scheme and MI-IA scheme respectively, to mitigate the cross-tier interference and the inter picocell interference in the transceiver design for picocells.

\subsubsection{ZF-IA based transceiver design scheme}

The key idea of the proposed ZF-IA based transceiver design for picocells can be summarized as follows: Firstly, the cross-tier interference is nullified by the interference suppression matrices of pico UEs. And then the inter picocell interference is removed by the precoding matrices of the pico BSs.

1) Design of the interference suppression matrices for pico UEs

The cross-tier interference is nullified by the interference suppression matrix of pico UE $l$ ( $\forall l \in\{1, \cdots, L\}$ ), so the condition $\mathbf{U}_{l}^{H} \mathbf{H}_{l, M} \mathbf{V}_{M}=\mathbf{0}$ must be satisfied. That is, $\mathbf{U}_{l}^{H}$ should lie in the left null space of $\mathbf{H}_{l, M} \mathbf{V}_{M}$, i.e.,

$$
\mathbf{U}_{l} \subseteq \operatorname{null}\left(\left(\mathbf{H}_{l, M} \mathbf{V}_{M}\right)^{\mathrm{H}}\right)
$$

The SVD of $\left(\mathbf{H}_{l, M} \mathbf{V}_{M}\right)^{\mathrm{H}}$ is

$$
\left(\mathbf{H}_{l, M} \mathbf{V}_{M}\right)^{\mathrm{H}}=\mathbf{U}_{l, M}\left[\begin{array}{cc}
\boldsymbol{\Sigma}_{l, M} & \mathbf{0} \\
\mathbf{0} & \mathbf{0}
\end{array}\right]\left[\begin{array}{ll}
\mathbf{V}_{l, M}^{(1)} & \mathbf{V}_{l, M}^{(0)}
\end{array}\right]^{\mathrm{H}}
$$

where $\mathbf{V}_{l, M}^{(0)}$ consists of the singular vectors corresponding to zero singular values of $\left(\mathbf{H}_{l, M} \mathbf{V}_{M}\right)^{\mathrm{H}}$. Thus the column vector of $\mathbf{V}_{l, M}^{(0)}$ is an orthogonal basis for the null space of $\left(\mathbf{H}_{l, M} \mathbf{V}_{M}\right)^{\mathrm{H}}$, i.e., $\left(\mathbf{H}_{l, M} \mathbf{V}_{M}\right)^{\mathrm{H}} \mathbf{V}_{l, M}^{(0)}=\mathbf{0}$, and then the interference suppression matrix of pico UE $l$ is

$$
\mathbf{U}_{l}^{\mathrm{H}}=\left(\mathbf{V}_{l, M}^{(0)}\right)^{\mathrm{H}}
$$

To guarantee the existence of the null space of $\left(\mathbf{H}_{l, M} \mathbf{V}_{M}\right)^{\mathrm{H}}, N_{R} \geq(K+1) d$ should be satisfied.

2) Design of the precoding matrices for pico BSs

The inter picocell interference is removed by the precoding matrices of the pico BSs. In order to cancel all the inter picocell interference, the following constraint must be satisfied

$$
\mathbf{U}_{l}^{\mathrm{H}} \mathbf{H}_{l, i} \mathbf{V}_{i}=\mathbf{0}, \forall i \in\{1, \cdots, L\}, i \neq l
$$


Thus $\mathbf{V}_{l}$ should lie in the null space of $\overline{\tilde{\mathbf{H}}}_{l}$, i.e., $\mathbf{V}_{l} \subset \operatorname{null}\left(\overline{\tilde{\mathbf{H}}}_{l}\right)$, where $\overline{\tilde{\mathbf{H}}}_{l}$ is defined as

$$
\overline{\tilde{\mathbf{H}}}_{l}=\left(\left(\mathbf{U}_{1}^{H} \mathbf{H}_{1, l}\right)^{\mathrm{T}}, \cdots,\left(\mathbf{U}_{l-1}^{H} \mathbf{H}_{l-1, l}\right)^{\mathrm{T}},\left(\mathbf{U}_{l+1}^{H} \mathbf{H}_{l+1, l}\right)^{\mathrm{T}}, \cdots,\left(\mathbf{U}_{L}^{H} \mathbf{H}_{L, l}\right)^{\mathrm{T}}\right)^{\mathrm{T}}
$$

The SVD of $\overline{\tilde{\mathbf{H}}}_{l}$ is

$$
\overline{\tilde{\mathbf{H}}}_{l}=\overline{\tilde{\mathbf{U}}}_{l}\left[\begin{array}{cc}
\overline{\tilde{\boldsymbol{\Sigma}}}_{l} & \mathbf{0} \\
\mathbf{0} & \mathbf{0}
\end{array}\right]\left[\begin{array}{cc}
\overline{\mathbf{V}}_{l}^{(1)} & \overline{\tilde{\mathbf{V}}}_{l}^{(0)}
\end{array}\right]^{\mathrm{H}}
$$

where $\overline{\tilde{\mathbf{V}}}_{l}^{(0)}$ consists of the singular vectors corresponding to zero singular values of $\overline{\tilde{\mathbf{H}}}_{l}$. Thus the column vector of $\overline{\tilde{\mathbf{V}}}_{l}^{(0)}$ is an orthogonal basis for the null space of $\overline{\tilde{\mathbf{H}}}_{l}$, i.e., $\overline{\tilde{\mathbf{H}}}_{l} \overline{\tilde{\mathbf{V}}}_{l}^{(0)}=\mathbf{0}$, and then the precoding matrix of pico BS $l$ is

$$
\mathbf{V}_{l}=\overline{\tilde{\mathbf{V}}}_{l}^{(0)}
$$

To ensure the existence of $d$ dimensions null space of $\overline{\tilde{\mathbf{H}}}_{l}, N_{P T} \geq L d$ should be satisfied. It should be noted that if we use the minimum number of receive antennas, i.e., $N_{R}=(K+1) d$, for the sake of $\mathbf{U}_{l}^{\mathrm{H}} \mathbf{H}_{l, M} \mathbf{V}_{M}=\mathbf{0}$, the column vector of $\mathbf{H}_{l, M} \mathbf{V}_{M}$ is an orthogonal basis for the null space of $\mathbf{U}_{l}^{\mathrm{H}}$. Moreover, $\mathbf{U}_{l}^{\mathrm{H}} \mathbf{H}_{l, i} \mathbf{V}_{i}=\mathbf{0}, \forall i \in\{1, \cdots, L\}, i \neq l$ is attained, thus $\mathbf{H}_{l, i} \mathbf{V}_{i} \prec \mathbf{H}_{l, M} \mathbf{V}_{M}$ is guaranteed. That is, the inter picocell interference to the pico UE $l$ is aligned onto the space of cross-tier interference. The procedure of ZF-IA based transceiver design for picocells is shown in Table 2.

Table 2. The procedure of ZF-IA based transceiver design for picocells

Step 1. The pico UE $l(\forall l \in\{1, \cdots, L\})$ estimates the interfering CSI, i.e., $\mathbf{H}_{l, j}(\forall j=\{1, \cdots, L, M\}, j \neq l)$ using pilots or a preamble, then they feed back the CSI to the macro BS through a dedicated common control channel.

Step 2. The interference suppression matrices for pico UEs are designed according to (12).

Step 3. The precoding matrices for pico BSs are designed using (16).

Step 4. The macro BS transmits the design matrices to each corresponding pico BS (or UE) by the backhaul link (or the downlink channel).

\subsubsection{ODC-IA based Transceiver Design Scheme}

The ZF-IA scheme can mitigate interference effectively but has not considered the CSI of the desired channel of pico UEs, which has a great impact on the data rate of pico UEs. In this section, we propose an ODC-IA scheme, which takes the CSI of the desired channel of pico UEs into account. That is, we add a constraint to guarantee the largest channel gain of the desired signal of pico UEs in the alignment processing, and then the data rate of the system will be further improved. 
It should be noted that the only difference between the proposed ODC-IA scheme and the ZF-IA scheme is the precoding matrices of pico BSs. Above all, in the ODC-IA scheme, the precoding matrices of pico BSs are designed for aligning the inter picocell interference to the pico UE $l$ onto the space of the cross-tier interference produced by the macro BS, i.e., $\mathbf{H}_{l, j} \mathbf{V}_{j} \prec \mathbf{H}_{l, M} \mathbf{V}_{M}(\forall j \in\{1, \cdots, L\}, j \neq l)$ should be fulfilled. And then, the precoding matrices for the pico BS $l \mathbf{V}_{l}$ should satisfy

$$
\begin{aligned}
& \mathbf{H}_{1, l} \mathbf{V}_{l} \prec \mathbf{H}_{1, M} \mathbf{V}_{M} \\
& \vdots \\
& \mathbf{H}_{l-1, l} \mathbf{V}_{l} \prec \mathbf{H}_{l-1, M} \mathbf{V}_{M} \\
& \mathbf{H}_{l+1, l} \mathbf{V}_{l} \prec \mathbf{H}_{l+1, M} \mathbf{V}_{M} \\
& \vdots \\
& \mathbf{H}_{L, l} \mathbf{V}_{l} \prec \mathbf{H}_{L, M} \mathbf{V}_{M}
\end{aligned}
$$

The inequality $N_{P T} \geq(L-1) N_{R}$ is a sufficient condition for solvability of (17). In other words, if $N_{P T} \geq(L-1) N_{R}$ is satisfied, $\left[\mathbf{H}_{1, l}^{\mathrm{T}} \cdots, \mathbf{H}_{l-1, l}^{\mathrm{T}}, \mathbf{H}_{l+1, l}^{\mathrm{T}} \cdots, \mathbf{H}_{L, l}^{\mathrm{T}}\right]^{\mathrm{T}}$ can be a full row rank matrix and $\quad \mathbf{V}_{l} \prec \tilde{\mathbf{V}}_{M} \quad$ is obtained, where $\quad \tilde{\mathbf{V}}_{M}=\left(\left[\mathbf{H}_{1, l}^{\mathrm{T}}, \cdots, \mathbf{H}_{l-1, l}^{\mathrm{T}}, \mathbf{H}_{l+1, l}^{\mathrm{T}}, \cdots, \mathbf{H}_{L, l}^{\mathrm{T}}\right]^{\mathrm{T}}\right)^{\dagger}$. $\left[\mathbf{H}_{1, M}^{\mathrm{T}}, \cdots, \mathbf{H}_{l-1, M}^{\mathrm{T}}, \mathbf{H}_{l+1, M}^{\mathrm{T}}, \cdots, \mathbf{H}_{L, M}^{\mathrm{T}}\right]^{\mathrm{T}} \mathbf{V}_{M}$ means that if arbitrarily d column vectors of the matrix $\tilde{\mathbf{V}}_{M}$ are selected as $\mathbf{V}_{l}$, the inter picocell interference can be aligned onto the space of cross-tier interference at $\mathrm{UE} j(\forall j \in\{1, \cdots, L\}, j \neq l)$.

In the following, the ergodic method is employed for searching $\mathbf{V}_{l}$ to guarantee the largest channel gain of the desired signal of pico UE $l$. We arbitrarily choose $d$ column vectors of the matrix $\tilde{\mathbf{V}}_{M}$ as $\mathbf{V}_{i}$, if $\left\|\mathbf{U}_{l}^{\mathrm{H}} \mathbf{H}_{l, l} \mathbf{V}_{i}\right\|_{2} \geq\left\|\mathbf{U}_{l}^{\mathrm{H}} \mathbf{H}_{l, l} \overline{\mathbf{V}}_{i}\right\|_{2}$, then $\mathbf{V}_{l}=\mathbf{V}_{i}$, where $\overline{\mathbf{V}}_{i}$ is the matrix composed by any $d$ columns of $\tilde{\mathbf{V}}_{M}$ except $\mathbf{V}_{i}$. Otherwise, another $d$ column vectors should be reselected and compared again until $\mathbf{V}_{l}$ is picked. The procedure of ODC-IA based transceiver design for picocells is shown in Table 3.

Table 3. The procedure of ODC-IA based transceiver design for picocells

Step 1. The pico UE $l(\forall l \in\{1, \cdots, L\})$ estimates the local CSI, i.e., $\mathbf{H}_{l, j}$ $(\forall j=\{1, \cdots, L, M\})$ using pilots or a preamble, then they feed back the CSI to the macro BS through a dedicated common control channel.

Step 2. The interference suppression matrices for pico UEs are designed according to (12).

Step 3. The precoding matrices for pico BSs are designed using the ergodic method which is mentioned above.

Step 4. The macro BS transmits the design matrices to each corresponding pico BS (or UE) by the backhaul link (or the downlink channel).

\subsubsection{MI-IA based Transceiver Design Scheme}

The ZF-IA and ODC-IA schemes can obtain a high data rate but require numerous receive antennas for the alignment processing. In this section, we relax the alignment constraint 
compared with ZF-IA and ODC-IA schemes. By sacrificing a little gain of the total achievable rate, the proposed MI-IA scheme can reduce the requirement of the receive antennas number.

The key idea of the proposed MI-IA based transceiver design for picocells can be summarized as follows: Firstly, we design the precoding matrices for pico BSs, aligning the inter picocell interference onto the space of the strongest cross-tier interference produced by macro BS. And then the aligned interference is nullified by the interference suppression matrices of pico UEs

1) Design of the precoding matrices for pico BSs

Define the certain macro UE as $l_{m}$, which receives the signal from BS $M$ producing the strongest cross-tier interference to UE $l$. That is, $l_{m}=\underset{k \in\{L+1, \cdots, L+K\}}{\arg \max }\left\|\mathbf{H}_{l, M} \mathbf{V}_{k}\right\|_{2}$. Align the inter picocell interference to UE $l$ onto the column space of $\mathbf{H}_{l, M} \mathbf{V}_{l_{m}}$, which is expressed as

$$
\begin{gathered}
\operatorname{span}\left(\mathbf{H}_{l, 1} \mathbf{V}_{1}\right)=\operatorname{span}\left(\mathbf{H}_{l, M} \mathbf{V}_{l_{m}}\right) \\
\vdots \\
\operatorname{span}\left(\mathbf{H}_{l, l-1} \mathbf{V}_{l-1}\right)=\operatorname{span}\left(\mathbf{H}_{l, M} \mathbf{V}_{l_{m}}\right) \\
\operatorname{span}\left(\mathbf{H}_{l, l+1} \mathbf{V}_{l+1}\right)=\operatorname{span}\left(\mathbf{H}_{l, M} \mathbf{V}_{l_{m}}\right) \\
\vdots \\
\operatorname{span}\left(\mathbf{H}_{l, L} \mathbf{V}_{L}\right)=\operatorname{span}\left(\mathbf{H}_{l, M} \mathbf{V}_{l_{m}}\right)
\end{gathered}
$$

So the precoding matrix of BS 1 should satisfy the following conditions,

$$
\begin{gathered}
\operatorname{span}\left(\mathbf{H}_{1, l} \mathbf{V}_{l}\right)=\operatorname{span}\left(\mathbf{H}_{1, M} \mathbf{V}_{1_{m}}\right) \\
\vdots \\
\operatorname{span}\left(\mathbf{H}_{l-1, l} \mathbf{V}_{l}\right)=\operatorname{span}\left(\mathbf{H}_{l-1, M} \mathbf{V}_{l-1_{m}}\right) \\
\operatorname{span}\left(\mathbf{H}_{l+1, l} \mathbf{V}_{l}\right)=\operatorname{span}\left(\mathbf{H}_{l+1, M} \mathbf{V}_{l+1_{m}}\right) \\
\vdots \\
\operatorname{span}\left(\mathbf{H}_{L, l} \mathbf{V}_{l}\right)=\operatorname{span}\left(\mathbf{H}_{L, M} \mathbf{V}_{L_{m}}\right)
\end{gathered}
$$

Then, a solution of (19) is obtained as

$$
\mathbf{V}_{l}=\left[\begin{array}{c}
\mathbf{H}_{1, l} \\
\vdots \\
\mathbf{H}_{l-1, l} \\
\mathbf{H}_{l+1, l} \\
\vdots \\
\mathbf{H}_{L, l}
\end{array}\right]^{\dagger}\left[\begin{array}{c}
\mathbf{H}_{1, M} \mathbf{V}_{1_{m}} \\
\vdots \\
\mathbf{H}_{l-1, M} \mathbf{V}_{l-1_{m}} \\
\mathbf{H}_{l+1, M} \mathbf{V}_{l+1_{m}} \\
\vdots \\
\mathbf{H}_{L, M} \mathbf{V}_{L_{m}}
\end{array}\right]
$$

To ensure the solution of (19) exists, $N_{P T} \geq(L-1) N_{R}$ should be satisfied.

2) Design of the interference suppression matrices for pico UEs

After designing the precoding matrices for pico BSs, most of the interference is aligned onto the column space of $\mathbf{H}_{l, M} \mathbf{V}_{l_{m}}$, and the rest interference is relatively weak, so the interference suppression matrix can be designed as 


$$
\mathbf{U}_{l} \subseteq \operatorname{null}\left(\left[\mathbf{H}_{l, M} \mathbf{V}_{l_{m}}\right]^{\mathrm{H}}\right)
$$

Denote the SVD of $\left[\mathbf{H}_{l, M} \mathbf{V}_{l_{m}}\right]^{\mathrm{H}}$ as

$$
\left[\begin{array}{ll}
\mathbf{H}_{l, M} \mathbf{V}_{l_{m}}
\end{array}\right]^{\mathrm{H}}=\mathbf{U}_{l}\left[\begin{array}{cc}
\boldsymbol{\Sigma}_{l} & \mathbf{0} \\
\mathbf{0} & \mathbf{0}
\end{array}\right]\left[\begin{array}{ll}
\mathbf{V}_{l}^{(1)} & \mathbf{V}_{l}^{(0)}
\end{array}\right]^{\mathrm{H}}
$$

The column vector of $\mathbf{V}_{l}^{(0)}$ is an orthogonal basis for the null space of $\left[\mathbf{H}_{l, M} \mathbf{V}_{l_{m}}\right]^{\mathrm{H}}$, so the interference suppression matrix can be designed as

$$
\mathbf{U}_{l}^{H}=\left(\mathbf{V}_{l}^{(0)}\right)^{\mathrm{H}}
$$

According to (21), the requirement of the receive antennas number is reduced to $N_{R} \geq 2 d$. The procedure of MI-IA based transceiver design for picocells is shown in Table 4.

Table 4. The procedure of MI-IA based transceiver design for picocells

Step 1. The pico UE $l(\forall l \in\{1, \cdots, L\})$ estimates the interfering CSI, i.e., $\mathbf{H}_{l, j}(\forall j=\{1, \cdots, L, M\}, j \neq l)$ using pilots or a preamble, then they feed back the CSI to the macro BS through a dedicated common control channel.

Step 2. The precoding matrices for pico BSs are designed using(20)

Step 3. The interference suppression matrices for pico UEs are designed according to (23).

Step 4. The macro BS transmits the design matrices to each corresponding pico BS (or UE) by the backhaul link (or the downlink channel).

\section{Complexity Analysis and Simulations}

\subsection{Complexity analysis}

The complexity of the proposed IA based transceiver design for macro cell mainly depends on the complexity of SVD scheme. For a given $m \times n$ matrix, the required arithmetic operations to determine the singular values and the corresponding singular vectors are given by $O\left(\min \left\{m n^{2}, m^{2} n\right\}\right)[10]$. The proposed IA based transceiver design for macro cell contains the arithmetic operations as follows: $K$ times SVD of a $(K-1) N_{R} \times N_{M T}$ matrix, the complexity of which is $O\left(K^{3} N_{M T} N_{R}^{2}\right) ; K$ times SVD of a $N_{R} \times\left[N_{M T}-(K-1) N_{R}\right]$ matrix, the complexity of which is $O\left(K N_{M T} N_{R}^{2}\right) ; K$ times multiplications of a $N_{R} \times N_{M T}$ matrix and a $N_{M T} \times\left[N_{M T}-(K-1) N_{R}\right]$ matrix, the complexity of which is $O\left(K N_{R} N_{M T}^{2}\right) ; K$ times multiplications of a $N_{M T} \times\left[N_{M T}-(K-1) N_{R}\right]$ matrix and a $\left[N_{M T}-(K-1) N_{R}\right] \times d$ matrix, the complexity of which is $O\left(K N_{M T}^{2} d\right)$. Therefore, the complexity of the proposed IA based transceiver design for macro cell is $O\left(K^{3} N_{M T} N_{R}^{2}+K N_{R} N_{M T}^{2}+K N_{M T}^{2} d\right)$. 


\subsubsection{ZF-IA based Transceiver Design}

The proposed ZF-IA based transceiver design for picocells contains the arithmetic operations as follows: $L$ times multiplications of a $N_{R} \times N_{M T}$ matrix and a $N_{M T} \times K d$ matrix, the complexity of which is $O\left(K L N_{R} N_{M T} d\right)$; $L$ times SVD of a $N_{R} \times K d$ matrix, the complexity of which is $O\left(K^{2} L N_{R} d^{2}\right)$; $L(L-1)$ times multiplications of a $d \times N_{R}$ matrix and a $N_{R} \times N_{P T}$ matrix, the complexity of which is $O\left(L^{2} N_{R} N_{P T} d\right)$; $L$ times SVD of a $(L-1) d \times N_{P T}$ matrix, the complexity of which is $O\left(L^{3} N_{P T} d^{2}\right)$. Therefore, the complexity of the proposed ZF-IA based transceiver design for picocells is $O\left(K L N_{R} N_{M T} d+K^{2} L N_{R} d^{2}+L^{2} N_{R} N_{P T} d+L^{3} N_{P T} d^{2}\right)$.

As the feasibility conditions of ZF-IA scheme is $N_{M T} \geq K N_{R}, N_{P T} \geq L d$ and $N_{R} \geq(K+1) d$, we choose the minimal antenna configurations for the macro BS, each pico BS and each UE, i.e., $N_{M T}=K(K+1) d, N_{P T}=L d$ and $N_{R}=(K+1) d$. Therefore the complexity of the proposed ZF-IA based transceiver design for macro cell is $O\left(K^{7} d^{3}\right)$ and that for picocells is $O\left(\left(K^{4} L+K L^{3}\right) d^{3}\right)$, and then the complexity of the proposed ZF-IA scheme is $O\left(\left(K^{7}+K^{4} L+K L^{3}\right) d^{3}\right)$.

\subsubsection{ODC-IA based transceiver design scheme}

It should noted that in the ODC-IA scheme, the precoding matrices for pico BSs are designed according to the ergodic method, so the maximum number of arithmetic operations is considered for the complexity computing. The ODC-IA based transceiver design for picocells contains the arithmetic operations as follows: $L$ times multiplications of a $N_{R} \times N_{M T}$ matrix and a $N_{M T} \times K d$ matrix, the complexity of which is $O\left(K L N_{R} N_{M T} d\right) ; L$ times SVD of a $N_{R} \times K d$ matrix, the complexity of which is $O\left(K^{2} L N_{R} d^{2}\right) ; L$ times computing the Moore-Penrose inverse matrix of a $(L-1) N_{R} \times N_{P T}$ matrix, the complexity of which is $O\left(L^{3} N_{P T} N_{R}^{2}\right) ; L$ times multiplications of a $N_{P T} \times(L-1) N_{R}$ matrix and a $(L-1) N_{R} \times N_{M T}$ matrix, the complexity of which is $O\left(L^{2} N_{R} N_{M T} N_{P T}\right) ; L\left(\begin{array}{c}K d \\ d\end{array}\right)\left(1+\left(\begin{array}{c}K-1) d \\ d\end{array}\right)\right)$ times multiplications of a $N_{P T} \times N_{M T}$ matrix and a $N_{M T} \times d$ matrix, the complexity of which is $O\left(L K^{2} N_{P T} N_{M T} d^{3}\right)$; $\left(\begin{array}{c}K d \\ d\end{array}\right)\left(\begin{array}{c}(K-1) d \\ d\end{array}\right)$ times comparisons, the complexity of which is $O\left(K^{2} d^{2}\right)$. Therefore the complexity of the ODC-IA based transceiver design for picocells is $O\left(K L N_{R} N_{M T} d+L K^{2} N_{R} d^{2}+L^{3} N_{P T} N_{R}^{2}+L^{2} N_{R} N_{M T} N_{P T}+L K^{2} N_{P T} N_{M T} d^{3}\right)$.

As the feasibility conditions of ODC-IA scheme is $N_{M T} \geq K N_{R}, N_{P T} \geq(L-1) N_{R}$ and $N_{R} \geq(K+1) d$, we choose the minimal antenna configurations for the macro BS, each pico BS and each UE, i.e., $N_{M T}=K(K+1) d, N_{P T}=(K+1)(L-1) d$ and $N_{R}=(K+1) d$. Therefore the complexity of the ODC-IA based transceiver design for macro cell is $O\left(K^{7} d^{3}\right)$ and that for picocells is $O\left(\left(L^{4} K^{3}+L^{2} K^{5} d\right) d^{3}\right)$, and then the complexity of ODC-IA scheme is $O\left(\left(K^{7}+L^{4} K^{3}+L^{2} K^{5} d\right) d^{3}\right)$. 


\subsubsection{MI-IA based transceiver design scheme}

The MI-IA based transceiver design for picocells contains the arithmetic operations as follows: $L$ times computing the Moore-Penrose inverse matrix of a $(L-1) N_{R} \times N_{P T}$ matrix, the complexity of which is $O\left(L^{3} N_{P T} N_{R}^{2}\right) ; L(L-1)$ times multiplications of a $N_{R} \times N_{M T}$ matrix and a $N_{M T} \times d$ matrix, the complexity of which is $O\left(L^{2} N_{R} N_{M T} d\right) ; L$ times multiplications of a $N_{P T} \times(L-1) N_{R}$ matrix and a $(L-1) N_{R} \times d$ matrix, the complexity of which is $O\left(L^{2} N_{R} N_{P T} d\right)$; $L$ times SVD of a $N_{R} \times d$ matrix, the complexity of which is $O\left(L N_{R} d^{2}\right)$. Therefore the complexity of the MI-IA based transceiver design for picocells is $O\left(L^{3} N_{P T} N_{R}^{2}+L^{2} N_{R} N_{M T} d+L^{2} N_{R} N_{P T} d+L N_{R} d^{2}\right)$.

As the feasibility conditions of the MI-IA scheme is $N_{M T} \geq K N_{R}, N_{P T} \geq(L-1) N_{R}$ and $N_{R} \geq 2 d$, we choose the minimal antenna configurations for the macro BS, each pico BS and each UE, i.e., $N_{M T}=2 K d, N_{P T}=2(L-1) d$ and $N_{R}=2 d$. Therefore the complexity of the MI-IA based transceiver design for macro cell is $O\left(K^{4} d^{3}\right)$ and that for picocells is $O\left(\left(L^{4}+L^{2} K\right) d^{3}\right)$, and then the complexity of the MI-IA scheme is $O\left(\left(K^{4}+L^{4}+L^{2} K\right) d^{3}\right)$.

From the complexity analysis, we can obtain that the complexity of the ODC-IA scheme is largest as a reason of the ergodic method. However, if we luckily choose the right precoding matrices for pico BSs at the beginning of computation, the complexity will be largely reduced. In addition, the complexity of the MI-IA scheme is smallest, which is smaller than that of the EHIA scheme [16], i.e., $O\left(K^{3} L^{7} d^{4}+K^{4} d^{3}\right)$ and that of the ZF scheme [17], i.e., $O\left(\left(K^{4}+L^{3} K+L K^{3}+L^{4}\right) d^{3}\right)$.

\subsection{Simulations}

In this section, the performance of the proposed ZF-IA, ODC-IA and MI-IA based transceiver design schemes are evaluated through Monte-Carlo simulations. To analyze the performance gain, the proposed transceiver design schemes are compared with three popular IA schemes in HetNets: the HIA scheme [3], the EHIA scheme [16] and the ZF scheme [17]. Considering the OSG mode of downlink two-tier HetNets which consists of one macro cell with $K$ UEs and $L$ picocells with one UE each, the macro BS is equipped with $N_{M T}$ transmitting antennas, each pico BS is equipped with $N_{P T}$ transmitting antennas, each UE is equipped with $N_{R}$ receive antennas and receives $d$ independent streams from its corresponding BS. The channels in this simulation are independent and identically distributed complex Gaussian variables with zero mean and unit variance. In the simulation, the parameters configuration is described as $\left(L, K, N_{M T}, N_{P T}, N_{R}, d\right)$.

Fig. 4 illustrates the total achievable rate comparisons for the proposed transceiver design schemes with varying CRE offset $\alpha$ in the case of 2 macro UEs and 2 picocells, where the minimum antennas number that the schemes required is used. Then, the parameters configuration is as follows, for ZF-IA scheme: $(2,2,6,3,3,1)$; for ODC-IA scheme: $(2,2,6$, 3, $3,1)$; for MI-IA scheme: (2, 2, 4, 2, 2, 1). It is shown that for the proposed transceiver design schemes, the total achievable rate is improved along with $\alpha$. It is mainly due to the cross-tier interference from pico BSs to macro UEs decreases with $\alpha$, meanwhile ZF-IA and ODC-IA 
scheme eliminate the cross-tier interference from the macro BS to pico UEs which increases with $\alpha$. Even though MI-IA scheme only eliminates the interference in the space of the strongest cross-tier interference for pico UEs, for the reason that most of the interference has been removed, and the residual interference is relatively weak, which has little influence to the total achievable rate.

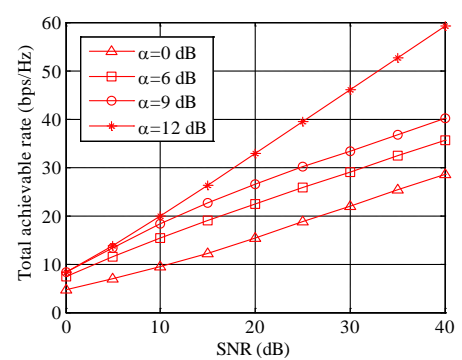

(a) ZF-IA scheme

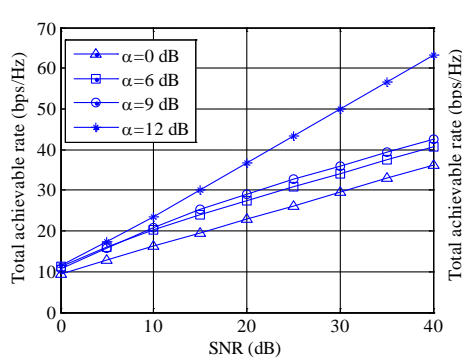

(b) ODC-IA scheme

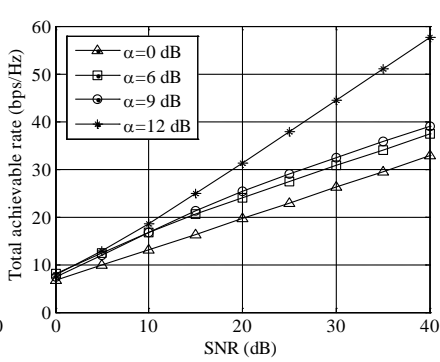

(c) MI-IA scheme

Fig. 4. A total achievable rate comparison with varying $\alpha$ in the case of 2 macro UEs and 2 picocells

Fig. 5 illustrates the total achievable rate comparisons for the proposed transceiver design schemes with varying parameters configuration when the CRE offset $\alpha=12 \mathrm{~dB}$. The comparisons have been done in three cases, where the minimum antennas number that the schemes required is used. That is, for ZF-IA scheme, case 1: (2, 2, 6, 3, 3, 1); case 2: (2, 3, 12, $2,4,1)$; case $3:(3,3,12,3,4,1)$. For ODC-IA scheme, case $1:(2,2,6,3,3,1)$; case $2:(2,3,12$, $4,4,1)$; case $3:(3,3,12,8,4,1)$. For MI-IA scheme, case $1:(2,2,4,2,2,1)$; case $2:(2,3,6,2$, $2,1)$; case $3:(3,3,6,4,2,1)$. It is shown that for each scheme, the total achievable rate is improved along with the number of macro UEs and picocells. As shown from the above parameters configuration, when the conditions of macro UEs, picocells and independent streams received by each UE are same, the requirement of antennas for MI-IA scheme is smallest, and that for ODC-IA scheme is largest. We then compare the total achievable rate with same parameters configuration when the CRE offset $\alpha=12 \mathrm{~dB}$ in Fig. 6, the minimum antennas number which satified the feasible condition of every proposed scheme is used as follows: case 1: $(2,2,6,3,3,1)$; case $2:(2,3,12,4,4,1)$; case $3:(3,3,12,8,4,1)$. It is shown from each case that the total achievable rate of ODC-IA scheme is largest and that of ZF-IA is smallest. And we can also see from each scheme that the total achievable rate is improved along with the number of macro UEs and picocells.

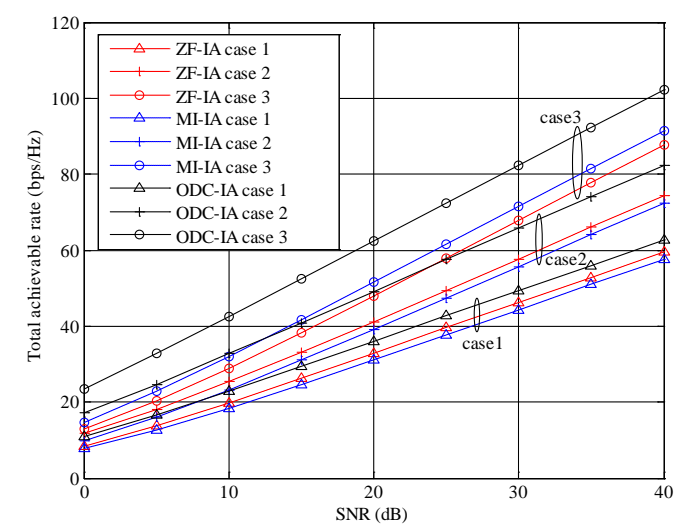

Fig. 5. A total achievable rate comparison with minimum parameters configuration 


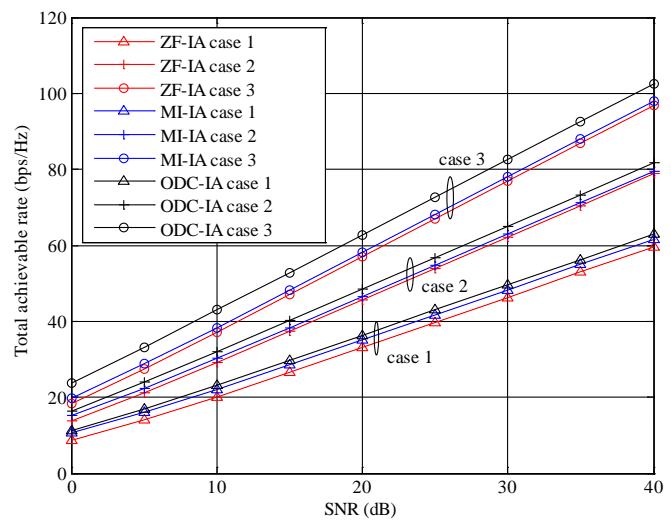

Fig. 6. A total achievable rate comparison with same parameters configuration

Fig. 7 illustrates a total achievable rate comparison for the proposed transceiver design schemes, the HIA scheme, the ZF scheme and the EHIA scheme with 2 macro UEs and 2 picocells when $\alpha=12 \mathrm{~dB}$. As the feasible condition of each scheme is different, the minimum antennas amount that each scheme required is used. Then the parameters configuration of each scheme is as follows: ZF-IA scheme: (2, 2, 6, 2, 3, 1); ODC-IA scheme: (2, 2, 6, 3, 3, 1); MI-IA scheme and HIA scheme: (2, 2, 4, 2, 2, 1); EHIA scheme and ZF scheme: (2, 2, 4, 3, 2, 1). In Fig. 8, a total achievable rate comparison for the proposed transceiver design schemes, the ZF scheme and the EHIA scheme with 3 macro UEs and 3 picocells is given. We set $\alpha=12 \mathrm{~dB}$, and the parameters configuration of each scheme is as follows: ZF-IA scheme: (3, 3, 12, 3, 4, 1); ODC-IA scheme: (3, 3, 12, 8, 4, 1); MI-IA scheme: (3, 3, 6, 4, 2, 1); EHIA scheme: (3, 3, 6, 7, 3, 1); ZF scheme: $(3,3,6,4,3,1)$. As shown in these figures, we can obtain that the total achievable rate of the proposed transceiver design schemes are larger than other schemes. The reason is that the proposed schemes are dedicated to the OSG mode, in which the number of interference channel links is smaller than that in CSG mode. Yet other schemes are proposed for the CSG mode, which bring in some costs to deal with the very weak interference when they are employed in the OSG mode, and then the performance is unsatisfactory. In addition, we can obviously see that the ODC-IA scheme can achieve the largest data rate. And in the case of 2 macro UEs and 2 picocells, the data rate of ZF-IA scheme is larger than that of MI-IA scheme, yet, the results are opposite in the case of 3 macro UEs and 3 picocells. It is mainly due to precoding matrices design for pico BSs. The IA scheme used in MI-IA scheme is more suitable to the situation of more picocells than the ZF scheme used in ZF-IA scheme.

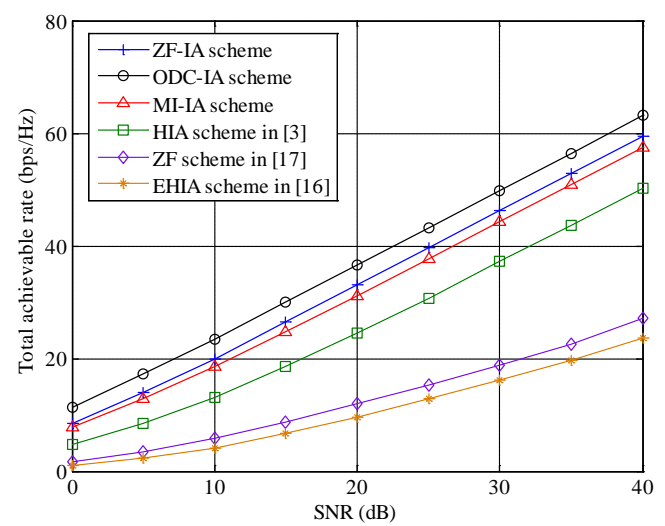

Fig. 7. A total achievable rate comparison with 2 macro UEs and 2 picocells 


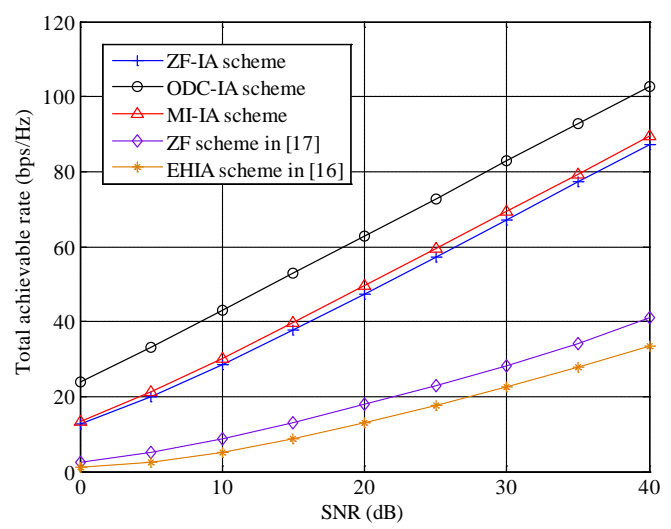

Fig. 8. A total achievable rate comparison with 3 macro UEs and 3 picocells

Fig. 9 illustrates the bit error ratio (BER) comparison for the proposed transceiver design schemes, the HIA scheme, the ZF scheme and the EHIA scheme with 2 macro UEs and 2 picocells. In the simulation, $\alpha=12 \mathrm{~dB}$; 64-QAM modulation and the minimum antennas amount that each scheme required is used. Then the parameters configuration of each scheme is as follows: ZF-IA scheme: (2, 2, 6, 2, 3, 1); ODC-IA scheme: (2, 2, 6, 3, 3, 1); MI-IA scheme and HIA scheme: (2, 2, 4, 2, 2, 1); EHIA scheme and ZF scheme: (2, 2, 4, 3, 2, 1). As shown in Fig. 9, the proposed schemes have better performance than other schemes. At $\mathrm{BER}=10^{-3}$, the ODC-IA scheme achieves approximately a $1 \mathrm{~dB}$ gain over the ZF-IA scheme, a $2 \mathrm{~dB}$ gain over the MI-IA scheme, a $6 \mathrm{~dB}$ gain over the HIA scheme, a $7 \mathrm{~dB}$ gain over the $\mathrm{ZF}$ scheme, and a 12dB gain over the EHIA scheme. It is obviously concluded that the ODC-IA scheme has the best BER performance, while the cost is high complexity.

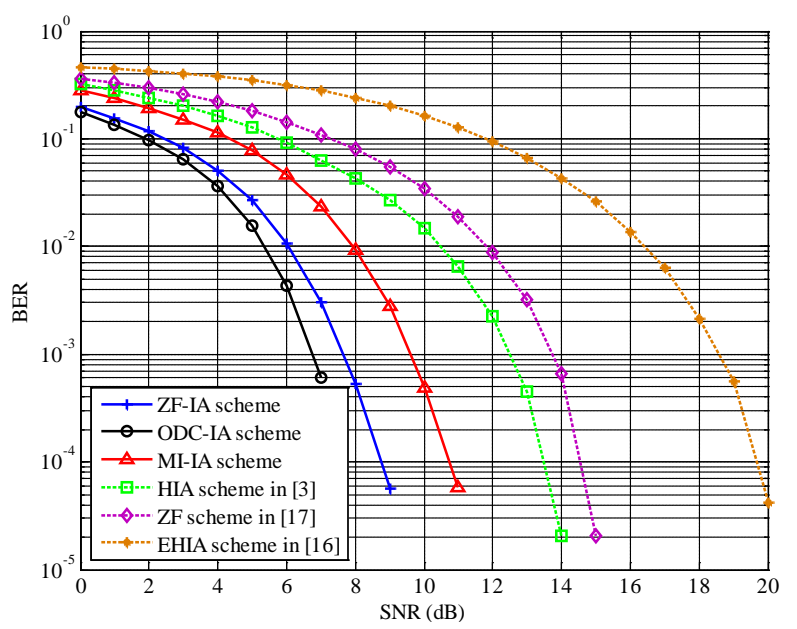

Fig. 9. The BER comparison with 2 macro UEs and 2 picocells

In Fig. 10, we present the total antenna usage comparison for the proposed transceiver design schemes, the ZF scheme and the EHIA scheme when the number of macro UEs $K$ is fixed and the CRE offset $\alpha=12 \mathrm{~dB}$ is set. Fig. 10(a) illustrates the case of $K=2$ and Fig. 10(b) illustrates the case of $K=3$. It is clearly observed that the total antenna usage for MI-IA scheme is less than that of ODC-IA scheme, and we can also see that the curve of the total antenna usage for the ZF-IA scheme has small slope, which is means that it has slower growth with $L$ than other schemes. 
Fig. 11 demonstrates the total antenna usage comparison for the proposed transceiver design schemes, the ZF scheme and the EHIA scheme when the number of picocells $L$ is fixed and the CRE offset $\alpha=12 \mathrm{~dB}$ is set, in which Fig. 11(a) illustrates the case of $L=2$, and Fig. 11(b) illustrates the case of $L=3$. As it can be seen, the total antenna usage for the ZF-IA scheme and the ODC-IA scheme increase non-linearly along with the number of macro UEs. That is, when $K$ is large, the requirement of antennas for these two schemes is larger than other schemes. However, the total antenna usage of the MI-IA scheme is smallest as expected.

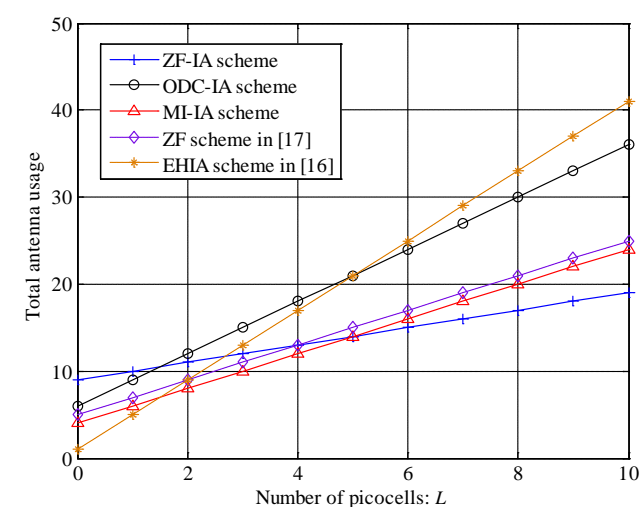

(a) $K=2$

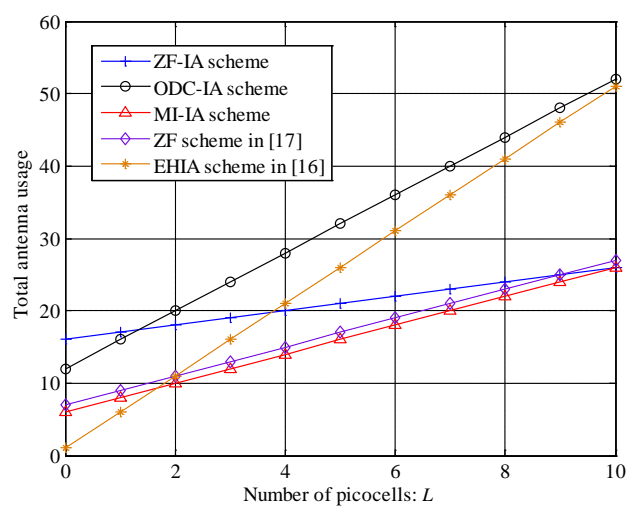

(b) $K=3$

Fig. 10. The total antenna usage comparison for the proposed for the proposed transceiver design schemes, the ZF scheme [17] and the EHIA scheme [16] when $K$ is fixed

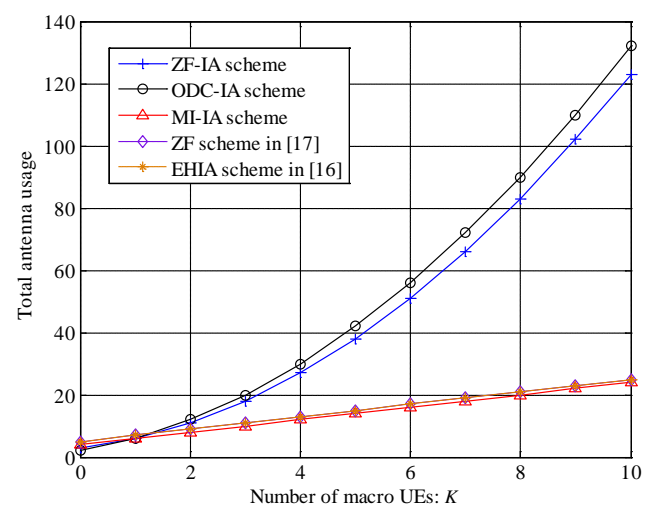

(a) $L=2$

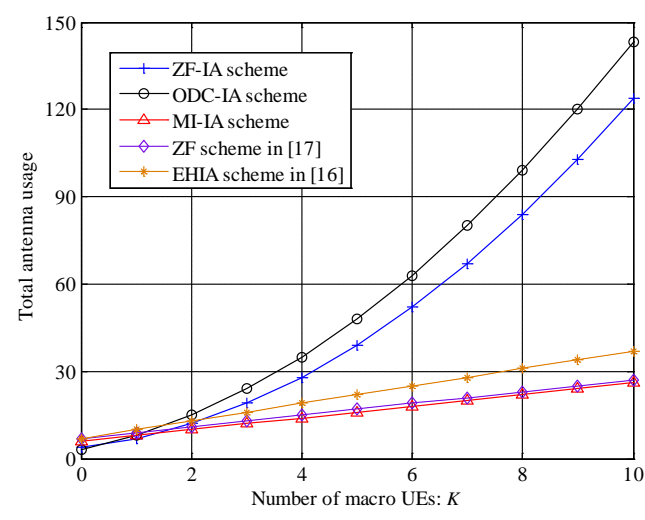

(b) $L=3$

Fig. 11. The total antenna usage comparison for the proposed for the proposed transceiver design schemes, the ZF scheme [17] and the EHIA scheme [16] when $L$ is fixed

\section{Conclusion}

In this paper, three IA based transceiver design schemes are proposed in the OSG mode of HetNets. For macro cell, we uniformly use BD scheme to eliminate the inter macro-user interference. And for picocells, three IA schemes are employed to mitigate the cross-tier interference and the inter picocell interference. The ZF-IA scheme, aligning the inter picocell interference onto an arbitrary sub-space of the cross-tier interference using ZF scheme, can eliminate interference effectively. Furthermore, considering the CSI of the desired channel of pico UEs in the process of designing the precoding matrices for pico BSs, the ODC-IA scheme 
can further improve the data rate. In addition, the MI-IA scheme, aligning the inter picocell interference onto the space of the strongest cross-tier interference and then nullify the aligned interference only, can reduce the requirement of the receive antennas number. From complexity analysis and simulation results, we confirm that the proposed transceiver design schemes can outperform the existing IA schemes in the OSG mode of HetNets, and the MI-IA based transceiver design scheme can reduce the requirement of the receive antennas number with lower complexity.

\section{References}

[1] D. Lopez-Perez, I. Guvenc, G. de la Roche, M. Kountouris, T. Quek, and J. Zhang, "Enhanced intercell interference coordination challenges in heterogeneous networks," IEEE Transactions on Wireless Communications, vol. 18, no. 3, pp. 22-30, 2011. Article (CrossRef Link)

[2] P. Lin, J. Zhang, Y. Chen, and Q. Zhang, "Macro-femto heterogeneous network deployment and management: from business models to technical solutions," IEEE Transactions on Wireless Communications, vol. 18, no. 3, pp. 64-70, 2011. Article (CrossRef Link)

[3] W. Shin, N. Lee, W. Noh, H.-H. Choi, B. Clerckx, C. Shin, and K. Jang, "Hierarchical interference alignment for heterogeneous networks with multiple antennas," in proc. of IEEE International Conference on Communications workshops (ICC workshops), pp. 1-6, 2011. Article (CrossRef Link)

[4] W. Shin, W. Noh, K. Jang, and H.-H. Choi, "Hierarchical interference alignment for downlink heterogeneous networks,” IEEE Transactions on Wireless Communications, vol. 11, no. 12, pp. 4549-4559, 2012. Article (CrossRef Link)

[5] V. Cadambe, and S. Jafar, "Interference alignment and degrees of freedom of the K-user interference channel,” IEEE Transactions on Information Theory, vol. 54, no. 8, pp. 3425-3441, 2008. Article (CrossRef Link)

[6] H. Chae, K. Kim, R. Ran, and D. Kim, “A single feedback based interference alignment for three-user MIMO interference channels with limited feedback,” KSII Transactions on Internet and Information systems, vol. 7, no. 4, pp.692-710, 2013. Article (CrossRef Link)

[7] K. Gomadam, V. Cadambe, and S. Jafar, "A distributed numerical approach to interference alignment and applications to wireless interference networks," IEEE Transactions on Information Theory, vol. 57, no. 6, pp. 3309-3322, 2011. Article (CrossRef Link)

[8] S. Peng, Y. Liu, H. Chen, and Z. Kong, "A distributed numerical approach to interference alignment and applications to wireless interference networks,” KSII Transactions on Internet and Information systems, Vol. 8, no. 6, pp.1926-1945, 2014. Article (CrossRef Link)

[9] W. Shin, N. Lee, J. Lim, C. Shin, and K. Jang, " On the design of interference alignment scheme for two-cell MIMO interfering broadcast channels," IEEE Transactions on Wireless Communications, vol. 10, no. 2, pp. 437-442, 2011. Article (CrossRef Link)

[10] J. Tang, and S. Lambotharan, "Interference alignment techniques for MIMO multi-cell interfering broadcast channels,” IEEE Transactions on Communications, vol. 61, no. 1, pp. 164-174, 2013. Article (CrossRef Link)

[11] J.-S. Kim, “Antenna Configurations for MIMO Interference Alignment in Three-User Heterogeneous Systems,” IEEE Transactions on Vehicular Technology, pp.1-5, 2014. Article (CrossRef Link)

[12] F. Pantisano, M. Bennis, W. Saad, M. Debbah, and M. Latva-aho, "Interference alignment for cooperative femtocell networks: A game theoretic approach,” IEEE Transactions on Mobile Computing, vol. 12, no. 11, pp. 2233-2246, 2013. Article (CrossRef Link)

[13] F. Pantisano, M. Bennis, W. Saad and M. Debbah, "Cooperative interference alignment in femtocell networks,” in proc. of IEEE Global Telecommunications Conference (GLOBECOM), pp. 1-6, 2011. Article (CrossRef Link)

[14] B. Guler, and A. Yener, "Selective interference alignment for MIMO cognitive femtocell networks,” IEEE Journal on Selected Areas in Communications, vol. 32, no. 3, pp. 439-450, 2014. 
Article (CrossRef Link)

[15] S. Sharma, S. Chatzinotas, and B. Ottersten, "Interference alignment for spectral coexistence of heterogeneous networks,” EURASIP Journal on Wireless Communications and Networking, vol.1, no. 46, pp. 1-14, 2013. Article (CrossRef Link)

[16] H.-H. Lee, and Y.-C. Ko, "Linear transceiver design based on interference alignment for MIMO heterogeneous networks,” IEEE International Symposium on Personal Indoor and Mobile Radio Communications (PIMRC), pp. 1645-1650, 2012. Article (CrossRef Link)

[17] J. Jin, X. Gao, and X. Wang, "New interference alignment schemes for downlink MIMO heterogeneous networks," IEEE International Symposium on Personal, Indoor and Mobile Radio Communications (PIMRC Workshops), pp.215-219, 2013 Article (CrossRef Link)

[18] Q. Niu, Z. Zeng, T. Zhang, Q. Gao and S. Sun, "Interference Alignment and Bit Allocation in Heterogeneous Networks with Limited Feedback,” IEEE International Symposium on Wireless Personal Multimedia Communications (WPMC), pp.514-519, 2014. Article (CrossRef Link)

[19] T. Akitaya, and T. Saba, "Hierarchical multi-stage interference alignment for downlink heterogeneous networks," Signal and Information Processing Association Annual Summit and Conference (APSIPA), pp.1-5, 2013. Article (CrossRef Link)

[20] G. Liu, M. Sheng, X. Wang, Y. Li and J. Li, "Joint interference alignment and avoidance for downlink heterogeneous networks,” IEEE Communications Letters, vol. 18, no. 8, pp. 1431-1434, 2014. Article (CrossRef Link)

[21] G. Liu, M. Sheng, X. Wang, W. Jiao, Y. Li, and J. Li, "Interference Alignment for Partially Connected Downlink MIMO Heterogeneous Networks,” IEEE Transactions on Communications, vol.63, no.2, pp.551-564, 2015. Article (CrossRef Link)

[22] J. Minho, T. Maksymyuk, R. L. Batista, T. F. Maciel, A. L. F. de Almeida, and M. Klymash, "A survey of converging solutions for heterogeneous mobile networks," IEEE Transactions on Wireless Communications, vol.21, no.6, pp.54-62, 2014. Article (CrossRef Link)

[23] T. Kudo, and T. Ohtsuki, "Cell range expansion using distributed Q-learning in heterogeneous networks,” in Proc. of IEEE Vehicular Technology Conference (VTC Fall), pp.1-5, 2013. Article (CrossRef Link)

[24] J. Minho, Longzhe Han, N. D. Tan, and H. P. In, “A Survey: Energy Exhausting Attacks in MAC Protocols in WBANs,” Telecommunication Systems, vol 58, no. 2, pp. 153-164, 2015. Article (CrossRef Link)

[25] C.-S. Chiu, and C.-C. Huang, “An Interference Coordination Scheme for Picocell Range Expansion in Heterogeneous Networks," in Proc. of IEEE Vehicular Technology Conference (VTC Spring), pp.1-6, 2012. Article (CrossRef Link)

[26] M. Simsek, M. Bennis, A. Czylwik, "Dynamic Inter-Cell Interference Coordination in HetNets: A reinforcement learning approach," in proc. of IEEE Global Telecommunications Conference (GLOBECOM), pp.5446-5450, 2012. Article (CrossRef Link) 


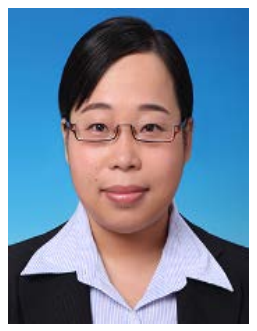

Qin Niu is currently pursuing the Ph.D. degree in Communication and Information Systems at Beijing University of Posts and Telecommunications (BUPT). Her current research field is in the areas of wireless communications, multiple-antenna technology, and interference management technology.

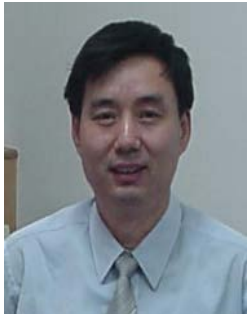

Zhimin Zeng is currently a professor in the School of Information and Communication Engineering and the chief director of Next Generation Communication System Theory and Technology Laboratory in Beijing University of Posts and Telecommunications (BUPT), Beijing, China. He received his Ph.D. degree in communications and information systems from BUPT. His current research interests include key technology of B3G/4G system, theory and technology of cognitive radio and network.

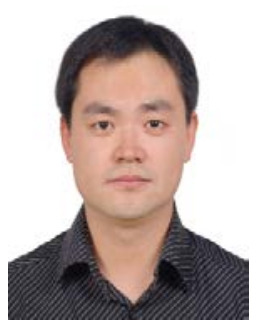

Tiankui Zhang BSc(Eng), PhD is a associate professor in the School of Informationand Communication Engineering of Beijing University of Posts and Telecoms(BUPT). He received his BEng and $\mathrm{PhD}$ degrees from BUPT, in the areas of Wireless Telecommunications in the years 2003 and 2008 respectively. His research field is in the areas of next generation wireless networks with particular focus on radio resource management and green radio.

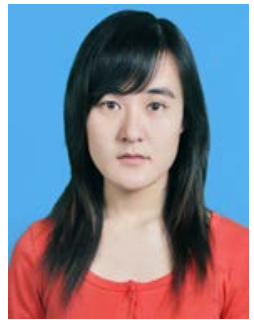

Zhirui Hu is currently pursuing the Ph.D. degree in Communication and Information Systems at Beijing University of Posts and Telecommunications (BUPT). Her current research field is in the areas of wireless communications, multiple-antenna technology, cooperative communications and signal processing technology. 